\title{
PLASTIC SURGERY UPDATE
}

\author{
By Mutaz B. Habal, M.D., F.A.A.P., F.I.C.S., F.R.C.S.(C), F.A.C.S. \\ Director of Tampa Bay Craniofacial Center/University of South Florida \\ Adjunct Professor of Human Sciences, Clinical Professor of Surgery, \\ Adjunct Professor of Material Science, University of Florida \\ Corresponding Member of the Dental Research Institute, UCLA.
}

\begin{abstract}
Based on a presentation at the Annual Spring Meeting of the Islamic Medical Association in Kissimmee, Florida. March, 1984.
\end{abstract}

\section{All Correspondence: Mutaz B. Habal, M.D., 4211 Carrollwood Village Drive, Tampa, Florida 33624, USA}

In the last decade plastic surgery has undergone a remarkable evolution and drastic changes. These changes are based on an understanding of newer concepts and newer principles, which have totally revolutionized the field of plastic and reconstructive surgery. The inpact of such principles and concepts have already spun off to other surgical specialties such as orthopedic surgery, hand surgery, urological surgery, and neurosurgery.

Historically, plastic surgenns have always evolved their specialty by developing newer tochniques and principles and passing them on to other surgical subspecialties. The best example of this is the development of skin flaps and skin grafting techniques for the full thickness graft which now almost every surgical specialist utilizes. The surgeon or specialist does not now have to be dependent on a plastic surgeon to apply these techniques. When recently asked, "how do you define plastic surgery in the 801 's?", the President of the American Society of Plastic and Reconstructive Surgery said "plastic surgery is sound clinical judgement, meticulous surgical techrique, and exploring bold, new frontiers. We are looking lorward in the 80's for development, and spread of these bold new frontiers."

What are these new frontiers? Basically the new frontiers can be listed as follows:

(1) Musculocutaneous flap surgery

(2) Microsurgery

(3) Craniofacial Surgery

(4) (Plastics) in Surgery

(5) Pediatric Plastic Surgery.

Musculocutaneous Flap Surgery

For thousands of years, plastic surgeons followed the dogma of Tagliacosi where he said, "when you do your naps, you should not touch the muscle," therefore all flap surgery and flap physiology was based on skin and fat Maps. However, Ralph Gurr of New York violated this old dogma when he demonstrated on his anatomical dissections that the blood-flow from the muscles to the skin is regional. Therefore by taking the muscle with the skin and fat producing a musculocutaneous nap, the blood Mow is preserved. This new surgical concept produced a complete evolution in the coverage of skin defects and utilization of flaps, particularly in the lower extremities. Most of the rotated, transposed, and advanced flaps depend on a basic vascular supply of the muscle. The muscle is the carrier of the skin and fal.

The basic idea of tlap surgery has been expounded in defects of the lower extremity which after trauma or tumor resection, result in amputation of the extremily. Now with most of these defects, flaps are utilized to complete the coverage of exposed or devitalized bone and the patients are able to walk on the reconstructed extremity without the use of any prosthesis. Other parts of the body, such as in head and neck surgery, upper extremity surgery, similar principles are utilized. Another area where musculocutaneous flap surgery has proved to be the must advantageous was in reconstruction of the breast after a radical mastectomy. A new frontier was also opened with the principle that the omentum can be moved on its. vascular supply and almost every muscle in the body which has a major or minor blood vessel can be used now as a rotation flap to be utilized for covierage of defects. The same muscle can be used by using microvascular techniques and transferred from one place to another in the body and the particular major blood supply is reconnected by microvascular anastrmosis. The muscle basically acts as a carrier and skin and fat is utilized for the coverage of the defect. The muscle that is transposed having reinuervation after transplantation will atrophy from disuse, but it will have served its purpose by the time it is completely atrophied, because the skin and fat used on the muscle will survive and producc complete coverage of the defect.

\section{Microsutgery}

Microsurgery is the art and science of utidization of an operating room microscope in the performance of a surgical procedure. The operating room microscope is used basically to see vessels or nerves that are not well visualized with the naked human eve. Through these techniques developed multiple specialties related to the field of microsurgery. In microvascular surgery, small vessels can now be moved and utilized to revascularize areas of the body for which, for many years the only treatment was amputation. Microneurosurgical utilization of the operating room microscope calls on the neurosurgeon or the neurovascular surgeon to visualize areas that were not visualized before and that enhances the safety of most operative procedures. At the same time nerves in neuro 
anastamosis became more delicate by utilization of the new principles. The Zeiss operating roon microscope series provides the state of the arl instrument for use in the operating roon. In plastic surgery, the OPMI 6-P-H and OPMI 7 are basically utilized in almost every hospital around the world. Other smaller cornpanies have introduced their operating room miscroscopes, but they do not reach the state of the art that the Zeiss has achieved.

Operating under magnification has also introduced a new era of surgical manipulation. The Zeiss series also utilizes rnagnifying glasses with wide angle lenses which are similar in power to those utilized in the microscope alone in the early 70 's.

Flaps now can be moved from one part of the body to the other. Nerves can be anastomosed, small vexsels can be put back together, tumors can be resected, and so fortl. As a result, instrumention has developed which can be well utilized while working under the microscope. Suture materials have been developed by the basic suture company, some of which are smaller than the human hair. All of these techniques produce a super specialization by themselves. A surgeon who is starting a practice, and is interested in using these basic principles in his practice, will have to spend extra time beyond an accredited residency to be able to understand and utilize these new techniques.

\section{Craniofacial Surgery}

Craniofacial surgery, as a new frontier, is now 15 years old. It was started basically by two surgeons in Paris and Buston, and now Centers around the country utilize their principle for this surgery. In brief, craniofacial surgery is surgery on the upper part of the orbit. By definition, craniofacial surgery means operating in and around the orbits, on the liard osseous frame work. The surgeon should be adepi in harvesting bone grafts and he should aiso be adept in utilizing intracranial as well as extracranial techniques. The intracranial techniques sometime roquire the assistance of a neurosurgeon and that neurosurgeon should be well experienced in handling pediatric neurosurgical problems, as similar problems dn arise in craniofacial surgery, because of the magnitude of these operations. The plastic surgeon should be prepared to have training beyond his accreclited residency so that he will be able to handle these complex problems. The impact of craniofacial surgery has been extremely strong on two aspects of plastic and reconstructive surgery. The first one is the impact on trauma and post traumatic defornities or what we call acquired deformities. Whercas the number of congenital malformations are decreasing, the number of acquired craniofacial deformities is increasing. Most of these patients will require transfer to a center for their treatment and a team is designated in each geographic area of the country to handle these difficult and intricate problems. The second vast impact that this surgery has is on cosmetic surgery.
Understanding the relationship between the osseous framc work and the soft tissues in the face allows the reconstructive surgeon to be able to reconstitute, pull. attach, and anchor various areas of the face that have sagged with gravity into the bony structure thus preventing those structures from reforming the acquired deformity that we see, particularly in elderly patients. New diagnostic measures have also been introduced and these diagnostic measures allow the surgeon to understand the deformity sufficiently to develop precise planning techniques prior to going into the operating room. Most of these techniques relate to the cumputerized demographic technicjues that are available either directly or by reformative computer assisted technology.

\section{(Plastics) in Surgery}

For quite some time plastics have been used in surgery and by plastics we mean biomalerials or materials that are inorganic. In 1984, there are still indications and contraindications to the use of such matcrials. There is no ideal biomaterial that can be used in the human body. but there are materials that come close enough to ideal, in the present state of the art, and can be utilized comfortably in patients undergoing reconstructive and plastic surgery. For exanple without the use of silicone gel bags, it would be extremely hard to reconstruct a patient, when an ablative procedure has been utilized to remove a breast, diseased with cancer.

Newer biomaterials are being introduced all the time, but because of the strict rules of the Federal Food and Drug Administration, far fewer new materials will be introdnced in the forthcoming ten years. Most likely some of the existing biomaterials will be refined and reintroduced again. Use of combination of grafts, which means utilizing the biomaterial to contour. act as a carrier, or completely fill a sjace of a missing part in the human body is with us to stay. Unfortunately, this is an area which is often abused. It is very hard at this point to convince the practicing surgeron that he cannot just walk to a shelf and pick up material and put it into the human body. There are indications and contraindications to the use of these materials. Understanding the principle of biomaterials, understanding the biology, the biophysios of these materials by the reconstructive surgeon is far more important than just making a simple cut and placing the materials into the human body.

\section{Pediatric Plastic Surgery}

Pediatric plastic surgery is emerging as a field by itself. It is very important for the surgeor to recognize childhood diseases, birth defects, abnomalities, tumors, and trauma.

Most of the children have to be treated in a different way than an adult. The physiology, the pathophysicology, the reactivity, the fluid requirements, and the anesthesia requirements are completely different. What has now emerged from this 
basic understanding is better rehabilitation, perhaps not only one more acceptable, but one that gives a far superior resuli than we achieved for many years previously. A pediatric plastic surgical approach to the problem gives a patient a more basic rehabilitative course that will allow the patient to get into the regular tract without any further abnormalities. Most of the pediatric plastic surgical problems, because they involve the patient's milestones, growth and development, achievements, and future understanding. necersitate the need for the parents to get involved directly with the rellabilitation. This team approach to the problem is emerging as a basic necessity in the $80^{\circ} \mathrm{s}$ when caring for pediatric patients, cspecially with facial deformities. In the patients who are born with a severe congenital deformity it is important to make the parents understand that the treatment does not end when the sutures are removed in the surgeon's oflice and the patient is discharged. Follow-up. menitoring growth and development is most important. Monitoring the patient's functional assesment is necessary every six months to a year in order to have proprer rehabilitory care for these children. The guided growth techniques in surgical manipulation are very important factors in the care of these patients; expecially the ones with facial deformities. In the guided growth, the paticnt's growth is assisted every few years surgically. Whenever lack of growth in certain areas is hampered greatly by a birth defect, radiation therapy, presence of a tumor, trauma, etc., surgical assistanec is required.

\section{Conclusion}

Thus to conclude: the field of plast ic and reconstructive surgery has undergone major changes in the $80^{\circ}$ s. The education of the plastic and reconstructive surgenn is an intricate part of nedical education. His approaches to the complicsted problems are extremely important. In the exlucation of the young generation of surgeons $: .2 . . .$. e ueveloping new careers, it is imperative to have piastic surgery as an intricate: part of thcir education. This to enable them to see the changes in plastic surgical techniques and in order for them to undersland and approach this complex problem rationally. This is also important so that they may understand the differences between a reconstructive surgeon, as the one who deals basically with the function and appearance, rather than one that deals with a life threatening situation, a situation which rarely. presents itself to the plastic and reconstructive surgeon. Rehabilitation and rolling back inlo the regular tracts in society, of a patient that needs plastic and reconstructive surgery, is as important as saving the life of a palient who has a life thrcatening situation. Interaction with the other specialties allows the plastic surgeons to formulate a team in all arpects of the surgery he deals with. 